\title{
Investigation of Online Corrosion Monitoring for Grounding Materials Based on Electromagnetic Ultrasonic Technology
}

\author{
Xiaolin $\mathrm{Li}^{1}$, Quan $\mathrm{Hu}^{2}$, Taishan $\mathrm{Hu}^{1}$, Bo Li ${ }^{2}$, Shangmao $\mathrm{Hu}^{1}$, Yi Zhang ${ }^{1}$ \\ ${ }^{1}$ Electric Power Research Institute, CSG, 11 Kexiang Road, Huangpu District, Guangzhou, China \\ ${ }^{2}$ Electric Power Research Institute of Guizhou Power Grid Co., Ltd., Guiyang, China
}

\begin{abstract}
Power grounding is a key aspect of ensuring safety in power facilities. However, the corrosion of grounding materials can cause accidents during power facility operation. Therefore, monitoring the corrosion status of grounding materials can eliminate hidden risks in the grounding network and ensure safe power operation. In this paper, electromagnetic ultrasonic thickness measurement technology was used to develop an online corrosion monitoring system for grounding materials via the installation of electromagnetic ultrasonic measurement probes on in-service power grounding materials. The results from a substation grounding network demonstrate that the online corrosion monitoring system can obtain more precise grounding corrosion data and has more extensive application prospects compared with other monitoring methods.
\end{abstract}

Keywords: Grounding, corrosion monitoring, electromagnetic ultrasonic technology

\section{Overview}

Grounding is a critical part of power facilities and is an important measure to ensure their safe operation. When a power facility fails or is struck by lightning, the fault current or the lightning current will flow into the earth through grounding, thereby ensuring the safety of electrical equipment and personnel. The materials used for grounding are usually metal materials such as steel, galvanized steel, copper, or copper-clad steel. Among them, galvanized steel has the highest usage. However, because these metal materials are buried in the ground, the soil acts as a corrosive medium and corrodes the metallic grounding materials. The corrosion behavior of power grounding materials is relatively complicated. In addition to electrochemical corrosion caused by the soil medium [1], the dispersion of current also promotes the corrosion speed of the grounding material when it is in an electrical environment [2]. Additionally, macrocell corrosion of the grounding grid [3,4], the use of grounding resistance-reducing agents, and galvanic corrosion between different metals all accelerate the corrosion of grounding materials. As a result, the actual service life of grounding networks on power transmission and transforming systems can be significantly lower than the designed life value. An investigation on the corrosion status of the grounding networks of selected substations in the provinces of Hunan, Guangdong, and Shaanxi in China showed that grounding networks that were in operation for 15-20 years suffered severe corrosion and multiple fractures. These results demonstrate that corrosion of galvanized steel grounding devices is a common phenomenon in China [5]. Corrosion also increases the grounding resistance of grounding devices and fractures block the path for fault current or lightning current in the grounding material. Consequently, power fault current and lightning current are unable to dissipate. Instead, the current will discharge in the equipment, causing equipment damage, power outages, and other power operation accidents as well as increasing the risk of severe casualties.

\section{Online corrosion monitoring for power grounding materials}

Ensuring the safe operation of power grounding is a systematic project, consisting of equipment and material design, maintenance, management, status monitoring, repair, and replacement as well as finding ground faults in time and

ISSN: 0010-8189

(c) CONVERTER 2020

www.converter-magazine.info 
eliminating the hidden dangers of power accidents. The corrosion status of grounding materials is a key factor affecting grounding performance. Monitoring the remaining thickness of a grounding material and variations in the corrosion rate combined with the power operation status can help with timely identification of problems in the grounding material, analysis of the grounding materials' corrosion status, identification of the cause of corrosion, and assist with taking measures to prevent electrical accidents. Monitoring corrosion rate is also an important factor in formulating management strategies and making decisions for power facilities. At present, the main methods for corrosion state monitoring and diagnosis of power grounding networks are the conductor resistance method, the electromagnetic field analysis method, and the corrosion monitoring method [6]. The resistance and electromagnetic methods consist of performing electrical and magnetic parameter tests on the grounding network and the use of mathematical methods to calculate defects in the grounding network to locate fault points. However, these two methods provide predicted results that can significantly differ from actual results due to the limitations of existing measurement and simulation technologies. It is impossible to directly obtain an accurate assessment of the grounding materials' loss and fault status through calculations and simulations by using relevant variations in resistance, potential, magnetic field, and electrochemical parameters. Because these parameters are affected by many factors, obtaining high-precision grounding loss results is extremely difficult.

In this paper, a direct in-situ method for monitoring the thickness variation of grounding materials is proposed. Based on electromagnetic ultrasonic thickness measurement technology, the grounding material can be directly monitored and thickness variation can be recorded in real time. The corrosion rate and variation in environmental corrosivity of the tested grounding material are recorded across a period of six months, providing timely feedback to monitor the occurrence of events that may cause grounding corrosion and thereby preventing electrical accidents.

\section{Principle and composition of online corrosion monitoring system for grounding materials}

Electromagnetic ultrasonic testing technology (TIMS-EMAT) is a new testing method that has developed rapidly in recent years. Thickness measurements are carried out according to the principle of ultrasonic pulse reflection. An AC coil is used to excite the eddy current on the outer wall of a metal pipe and then an external magnetic field is applied. The eddy current can generate ultrasonic waves on the outer wall of the tested pipe under the action of the Lorentz force, providing a method for non-contact ultrasonic testing. The surface of the measured material is regarded as a piezoelectric wafer, so no coupling medium is required and the surface of the measured material does not need special cleaning. Due to these features, EMAT technology is suitable for long-term continuous observation and measurement $[7,8]$.

In this work, an electromagnetic ultrasonic probe was placed on one side of grounded galvanized flat steel and sealed by resin. The grounded flat steel with the sealed probe was buried in the working environment with a test cable connected to the ground measurement control system. Measurement data were wirelessly transmitted from the ground measurement control system to a computer for storage, dealing, and analysis. Multiple electromagnetic ultrasonic probes were used to form a composite system and continuous corrosion monitoring was performed on different parts of the grounding network. Using high-speed data sampling technology, a measurement accuracy of $0.02 \mathrm{~mm}$ can be achieved for materials with a thickness of at least $3 \mathrm{~mm}$, meeting the corrosion monitoring requirements for power grounding materials. A schematic diagram of the EMAT-based online corrosion monitoring probe for grounding materials is shown in Figure 1.

ISSN: 0010-8189

(c) CONVERTER 2020

www.converter-magazine.info 


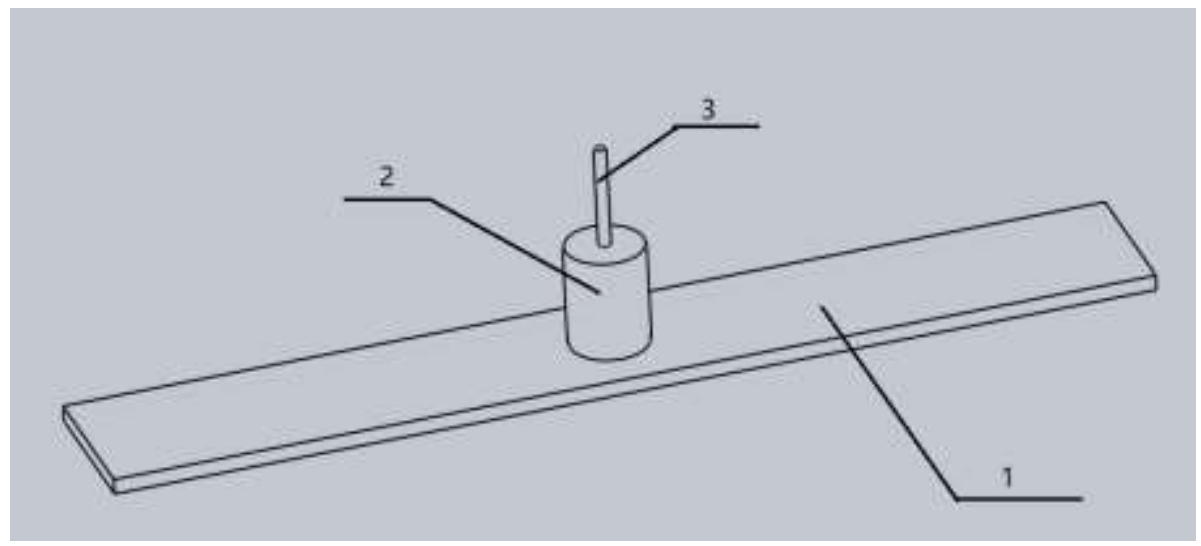

$\begin{array}{lll}\text { 1. Grounding galvanized flat steel } & \text { 2. Electromagnetic ultrasonic probe } & \text { 3. Probe test cable }\end{array}$

Fig 1: Schematic diagram of the EMAT-based online corrosion monitoring probe for grounding materials.

The ground measurement control system is responsible for controlling the system, taking measurements, logically managing the electromagnetic probe signal, data acquisition, data transmission, and data analysis. This system can is capable of long-term unattended automatic monitoring, analyzing environmental corrosivity and triggering an alarm based on corrosion speed. The ground measurement control system consists of the following components:

1) Ultrasonic measurement control unit: composed of a measurement control box and an ultrasonic thickness gauge.

2) Signal transmission unit: composed of wireless signal transmission devices.

3) Power control system: composed of solar panels, power control devices, and batteries.

4) Data analysis and processing software: for automatically storing data and performing calculations and analysis.

A flow chart of the ground measurement control system is shown in Figure 2.

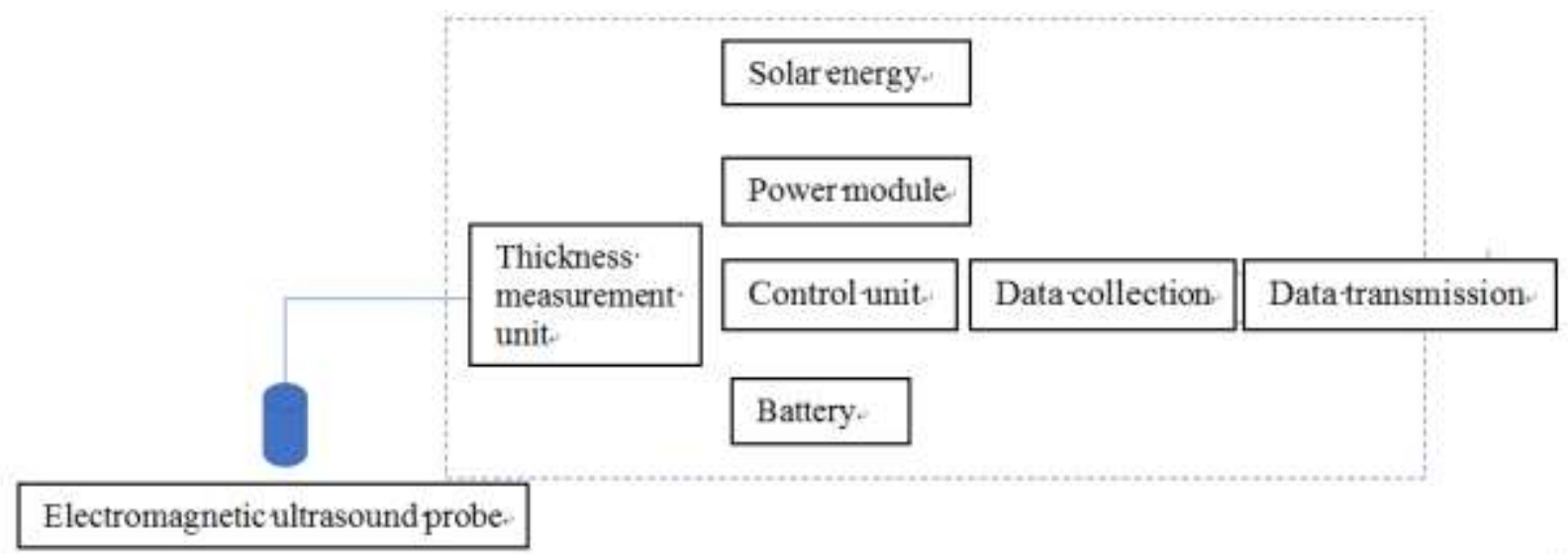

Fig 2: Schematic diagram of the composition of the ground measurement control system.

In-situ real-time thickness monitoring provides enough data for monitoring the corrosion rate of grounding materials in space and time. The corrosion rate of grounding materials across a period of time can be calculated by the following formula:

ISSN: 0010-8189

(c) CONVERTER 2020

www.converter-magazine.info 


$$
c_{\nabla t}=365 \times \frac{T_{t 1}-T_{t 2}}{t_{1}-t_{2}}
$$

Where:

$c_{\nabla t}$ : the corrosion rate of the grounding material across a specified period of time

$\mathrm{T}_{\mathrm{t} 1}$ : the thickness of the grounded galvanized flat steel at time $\mathrm{t} 1$

$\mathrm{T}_{\mathrm{t} 2}$ : the thickness of the grounded galvanized flat steel at time $\mathrm{t} 2$

\section{Application of online corrosion monitoring system for grounding materials in a power substation}

The online corrosion monitoring system based on EMAT technology was applied to different positions on the grounding network of a $66 \mathrm{kV}$ substation in Liaoning, China. Electromagnetic ultrasonic probes were installed on the grounding flat steel at four positions: under the main transformer, the switch, the steel structure, and the fence. Continuous monitoring was performed across a six-month period. Thickness measurements were recorded once per week.

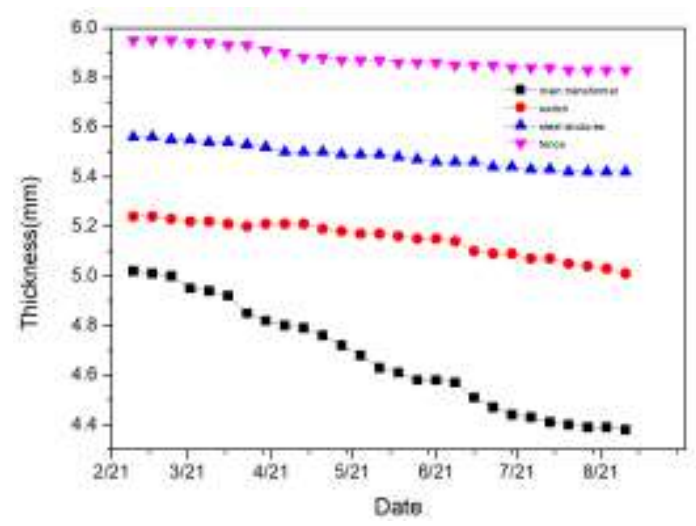

Fig 3: Thickness variation curve at different positions with over six months.

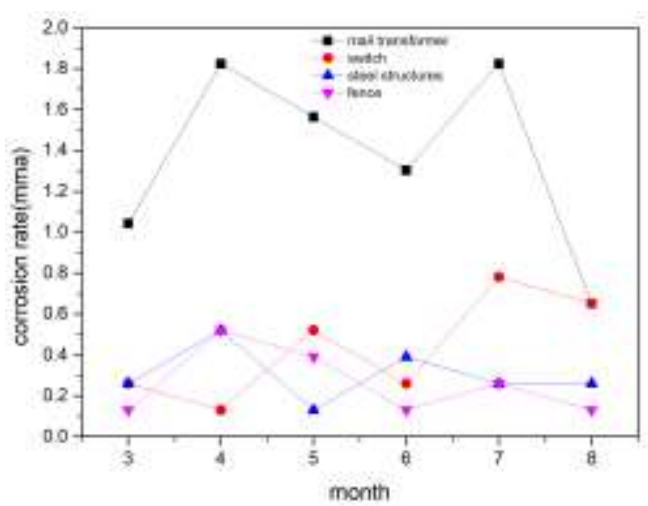

Fig 4: Monthly corrosion rate variation curve at different positions.

As can be seen from the variation curve of the grounding grid thickness across six months (Figure 3), the thickness of the grounding material under the main transformer decreased more rapidly than the thickness of ISSN: 0010-8189 
the grounding material in the other three positions. The monthly average corrosion rate of the grounding material at different positions calculated according to Formula 1 shows that the grounding material under the main transformer had a higher corrosion rate. This is because the grounding material under the main transformer must continuously withstand the corrosive effect of the transformer's working current and induced current. This demonstrates that corrosion rates of the grounding material in grounding networks can show significant differences across the network due to different working environments.

\section{Conclusions}

Electromagnetic ultrasonic technology has achieved excellent results in online corrosion monitoring of power grounding materials due to highly accurate non-contact measurements. This technology has wide application prospects in monitoring the corrosion of power grounding systems, providing early warning of ground faults, and assisting with fault diagnosis.

\section{References}

[1] K.H. Chen, "Study on the Corrosion Characteristics of Grounding Grid in Soil", Electric Porcelain Lightning Arrester, August, vol. 4, pp.39, 2009.

[2] X.M. Wu, "Study on Power Frequency In-Ground Current Corrosion of Carbon Steel Grounding Materials", Total Corrosion Control, Issue 12, 2014.

[3] X.W. Hu, "Research on Corrosion and Protection of Grounding Grids", Hubei Electric Power, vol. 26, no. 3, June, pp. 31, 2006.

[4] Y.Q, "Soil Corrosion of Grounding Electrodes", Environmental Technology, February, Issue 1, pp. 15, 2010.

[5] S. Song, "Corrosion and Protection of Power Grounding Device", China Electric Power Press, pp. 12, 2017.

[6] F.L. Li, "Research Progress on Grounding Grid Corrosion and Protection", Industrial Construction, vol, 42, supplement, pp. 783, 2012.

[7] J.H. Cao, G.B. Yan, X.F. Han, "Principle and Application of Electromagnetic Ultrasonic Thickness Measurement-A New Type of Ultrasonic Thickness Measurement Method", Journal of Zhejiang University (Engineering Science Edition), vol. 36, no. 1, pp. 88.

[8] T.H. Liu, X.L. Qiao, J.G. Chen, "System Design and Implementation of EMAT Ranging and Thickness Measurement", Measurement and Control Technology, vol. 24, no. 4, pp. 20, 2005.

ISSN: 0010-8189 